\title{
Gambling problems amongst the CALD population of Australia: hidden, visible or not a problem?
}

\author{
Matthew Stevens ${ }^{1,2^{*}}$ and Kate Golebiowska ${ }^{1}$
}

\author{
* Correspondence: matthew. \\ stevens@menzies.edu.au \\ ${ }^{1}$ Charles Darwin University, The \\ Northern Institute, Darwin, Australia \\ ${ }^{2}$ Charles Darwin University, Menzies \\ School of Health Research, Darwin, \\ Australia
}

\begin{abstract}
There have been mixed research results when studying gambling problems in Culturally and Linguistically Diverse (CALD) communities in Australia and internationally. This study tests the feasibility of using nationally representative General Social Surveys for examining trends and patterns in gambling problems and other life stressors amongst the Australian CALD population. Two surveys were analysed to determine whether the CALD population experienced gambling problems and other life stressors at different levels to the non-CALD population, and to identify, using multivariable models, whether CALD related variables showed evidence of an association with reported gambling problems after adjustment for other covariates. There was no evidence that 2002 estimates of gambling problems were different in CALD and non-CALD populations. In 2006, there was evidence that gambling problems were lower in the CALD population compared with the nonCALD population (1.3\% cf. 3.5\%). In 2002 multivariable models there was no evidence of an association between CALD status or related variables with gambling problems, after adjustment for other variables. In 2006 multivariable models, there was evidence of an association between being the CALD population (protective), and being born in Oceania or New Zealand (risk) with gambling problems, after adjustment for other variables.
\end{abstract}

Keywords: Culturally and linguistically diverse, Gambling, Population surveys, Ethnicity

\section{Background}

Australia has an ethnically diverse population, with nearly $25 \%$ of the population being born overseas (Department of Immigration and Citizenship 2009). Within this group, the term 'Culturally and Linguistically Diverse' (CALD), has been used to describe people born overseas who do not speak English at home. The make-up of this group within Australia has changed markedly since early immigration waves post World War II, with early waves of immigrants predominantly coming from the United Kingdom and European countries and an increase in intake from Asian countries from the 1980's onwards (Australian Bureau of Statistics 2008; Stevens et al. 2010).

Grants of permanent residence visas in Australia are made in three categories: skill (comprises business), family and humanitarian. Skilled and business migrants have been sought by successive Australian Governments predominantly since the 1980s to

\section{Springer}

(C) 2013 Stevens and Golebiowska; licensee Springer. This is an Open Access article distributed under the terms of the Creative Commons Attribution License (http://creativecommons.org/licenses/by/2.0), which permits unrestricted use, distribution, and reproduction in any medium, provided the original work is properly cited. 
contribute to the economic growth and development of the country. Their proportionate share has been increasing and in 2007-08 it represented nearly $70 \%$ of the annual migration program in Australia. The Australian Government periodically adjusts skilled entry criteria to select higher calibre migrants and match economic conditions. For example, in 2007 and 2010, it tightened entry requirements for skilled visas with regards to the types of skills, level of education and proficiency in English language (Minister for Immigration and Citizenship 2007, 2010), leading to more educated immigrants compared with past decades (Department of Immigration and Citizenship 2009). The policy changes and economic conditions between 1999-2000 and 2008-09, led to a more than doubling in the number of skilled and business visas from 38,000 to 134,000, respectively (Markus et al. 2009). The family stream group of migrants are not subject to skilled entry criteria and many of these visa holders are women from various Asian countries and the proportionate share of this stream has fallen from 42\% (200001) to $30 \%(2008-09)$ as the skilled and business stream increased its share (Department of Immigration and Citizenship 2009).

Australia has a long history of re-settling refugees. This is the smallest migration stream, which over the decade of 1999-2000 and 2008-09 has fluctuated between 10,000 and 14,000 annually. Around half of these places go to refugees selected from refugee camps (Markus, et al. 2009). Currently, humanitarian entrants largely come from the African, Asian and Middle East countries. Their human capital varies greatly from no schooling at all to trades and professional qualifications but no recognition of qualifications and common English language difficulties mean it is some time before this group can become financially self-sufficient. Humanitarian and family entrants are entitled to free English language tuition upon arrival to enable them to quickly participate in economic and social life of Australia. All in all, the composition of the Australian immigration intake is socio-economically and culturally very diverse, includes native and non-native English speakers as well as those who will learn it only in Australia.

Research on problem gambling with CALD populations has tended to focus on specific ethnic communities (Loo et al. 2008), or on particular geographic locations (Brozovic-Basic 2005; Cultural Partners Australia 2000; The Ethnic Communities' Council of New South Wales 1999) or both (Chui and O'Connor 2006). The research in Australia and from other English speaking countries has found that non-Caucasian ethnicity is a risk factor for gambling related harm (Clarke et al. 2007; Gibbs Van Brunschot 2000; Raylu and Oei 2004). Factors found to be conducive to gambling may be a) uniquely related to the minority status experience and b) to more universal circumstances (e.g. low income status) relevant to gambling among ethnic groups and the general community alike. Three cultural variables, which have been considered in the uptake and maintenance of gambling, are: a) adherence to cultural values; b) acculturation, and c) culturally-determined help seeking behaviours (Oei and Raylu 2009; Raylu and Oei 2004). These three factors have also been found to be important in the initiation of mental health and drug and alcohol-related issues (De La Rosa et al. 2000; Escobar et al. 2000; Loue 1998; Westermeyer 1999).

Cultural norms, practices and beliefs related to gambling can be passed to an individual in different ways. The social learning perspective, which proposes that such norms and beliefs are socially transmitted is often invoked (Bandura 1986). For example, the 
transfer can occur via the behaviour of the immediate family members and/or other respected community members who are perceived as role models. It can also occur if role models show their approval of gambling, or share an oral or written history which accepts it (Raylu and Oei 2004). Studies of the role of familial influence on gambling focus upon parental/caregiver gambling and they have found correlations between problem and/or pathological gambling of the offspring and parents' gambling (Ladouceur and Mireault 1988; Lesieur et al. 1991; Lesieur and Heineman 1988; Oei and Raylu 2009; Teo et al. 2007; Toneatto and Brennan 2002).

So, while there have been numerous small-scale, geographically-contained studies across Australia, there is a lack of data that are comparable through time that allow for identification of gambling problems at the national level for the CALD population. The Australia Bureau of Statistics (ABS) conducts the General Social Survey (GSS) 4-yearly, which collects a broad range of information from demographic, social, cultural, economic and emotional and social well-being (ESWB) domains including information on gambling problems. However, the feasibility of using this data set to measure gambling problems for Australia's CALD population has not been tested. The advantage of being able to use information collected from national surveys comes from the use of a consistent methodology to measure gambling problems geographically and over time.

To test the feasibility of using the routinely collected nationally representative surveys for measuring gambling problems amongst the CALD population, data access was obtained to analyse the 2002 and 2006 GSS's. This allowed for a range of important demographic, socioeconomic, and social connectedness variables to be included in the analyses of reported gambling problems amongst the CALD population. Specifically the research addresses the following questions:

(1) Does the CALD population experience life stressors, including gambling problems, at different levels to the non-CALD population in Australia?

(2) Are CALD status and related variables associated with reported gambling problems after adjustment for other variables in multivariable models?

(3) Is the GSS a suitable data set to measure gambling problems amongst the CALD population?

\section{Methods}

Data sources and survey design

Full details of sample design, collection methods, and data quality for the General Social Surveys (GSSs) have been reported elsewhere (Australian Bureau of Statistics 2003, 2007) and a summary is therefore provided here. The GSS is a general population survey conducted every four years and forms part of the ABS social survey program. The 2002 and 2006 GSSs employed a stratified multistage area sample, with a scope that included all people aged 15 years and over in non-remote areas of Australia, with all data collected in face-to-face interviews. The map in Figure 1 provides the boundaries of the remoteness structure used in Australian Bureau of Statistics (ABS) surveys in Australia. Non-remote areas include major cities and inner and outer regional areas, and cover over $98 \%$ of Australia's total population. The survey goes through a process of testing questions, before completing a full dress-rehearsal of all survey processes. 


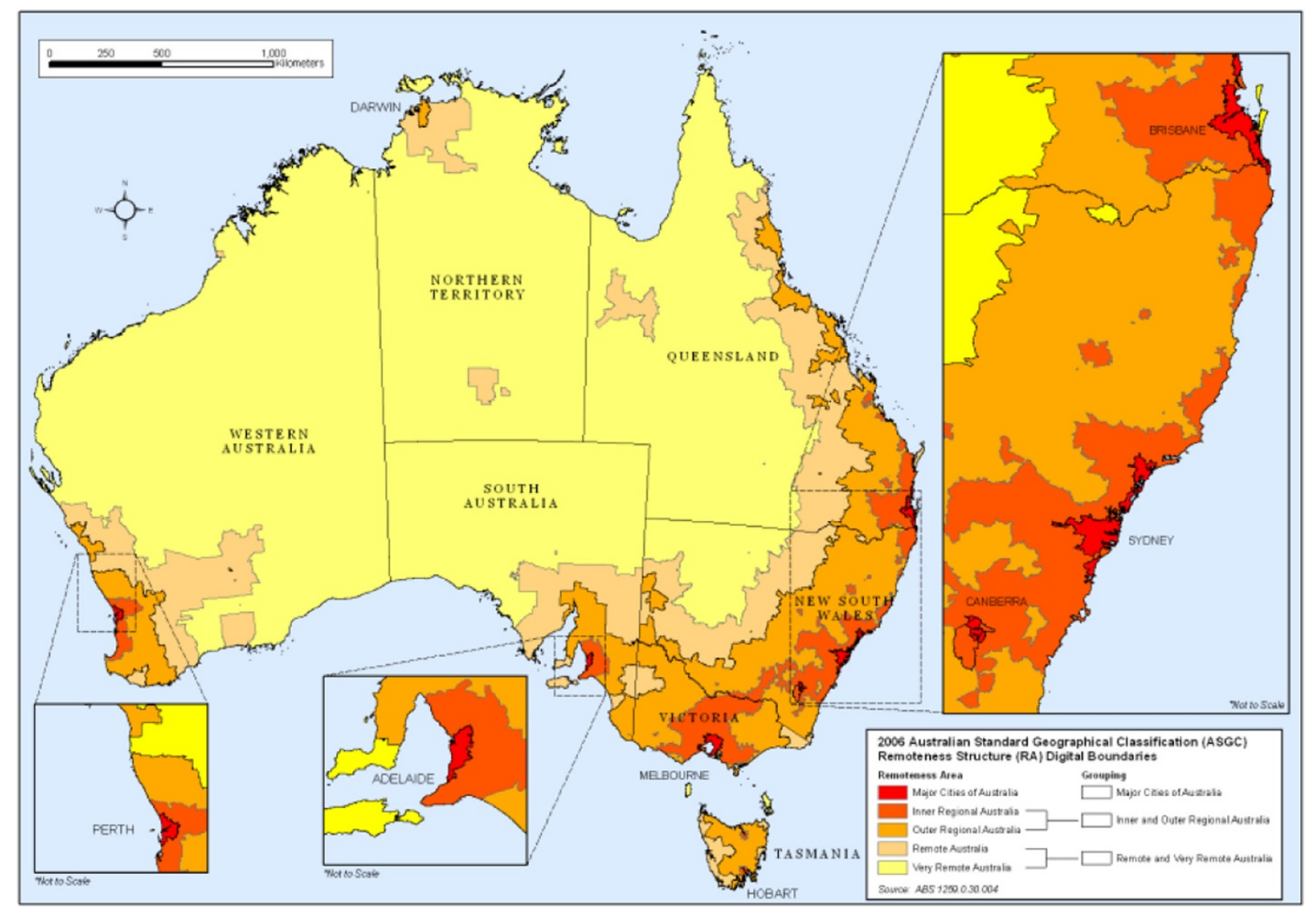

Figure 1 ABS Australian Standard Geographical Classification remoteness structure. 


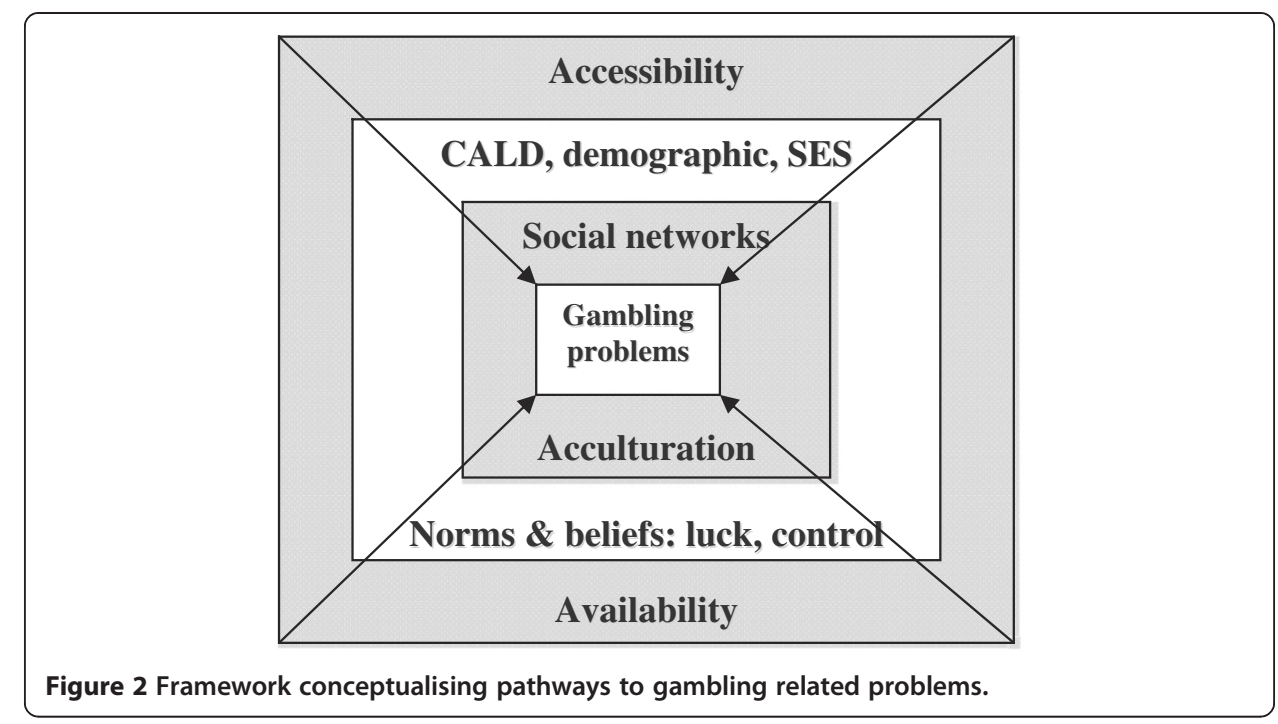

For the current analyses, only data from people aged 18 years and over were used because respondents under the age of 18 years were not administered the module containing the question on gambling problems. Confidentialised Unit Record Files (CURFs) or data files for the GSSs were accessed via the ABS Remote Access Data Laboratory or RADL (Australian Bureau of Statistics 2006). This is a web-based interface that allows the user to enter code, which is then processed on site at the ABS, to ensure confidentiality restrictions associated with the 1905 Census and Statistics Act for Australia are adhered to. All ABS survey data is de-identified and ethics approval was not required as the analyses constitute secondary use of data.

\section{Socio-environmental pathways model to gambling problems}

Figure 2 shows a conceptual framework representing pathways to gambling problems (from the outside square through to the middle square). Starting from the outside moving in, if gambling opportunities through accessibility or availability are not there then it is not possible to develop problems with gambling (Nerilee Hing and Haw 2009; Moore and Ohtsuka 1997). If gambling is accessible and available then factors relating to being a member of the CALD population along with other socio-demographic and socioeconomic factors may indicate higher (or lower) risk associated with developing gambling related problems (Stevens et al. 2010; Teo, et al. 2007; Volberg 1994). For example, young men, low or high income, and labour force status have all been shown to be associated with increased levels of problem gambling (Young and Stevens 2009). Also in this square are social norms and personal beliefs (e.g. luck and control over outcomes) which have been shown to have bearing on the risk of problem gambling (Chantal and Vallerand 1996; Nerilee Hing and Breen 2001; Tanasornnarong et al. 2004; Wohl and Enzle 2002; Wohl et al. 2005). For example, research suggests that gamblers who learnt to play from their parents are at higher risk due to the normalisation of gambling as an activity (even when it is problematic) (Breen et al. 2010). The box surrounding 'gambling problems' contains factors associated with social networks 
and acculturation. These factors can be either protective (Beattie et al. 1999; Stanton 2004) or risk factors (Kidman 2004) in the development of gambling problems.

\section{Measurement of gambling problems and the Negative Life Events Scale (NLES)}

The NLES is a regular survey module used by the ABS in social and health surveys, and is designed to measure individuals' emotional and social wellbeing (ESWB) by identifying life stressors that individuals are exposed to. The NLES module was developed for use with the Aboriginal and Torres Strait Islander populations, with the specific purpose of comparing ESWB between the Indigenous and non-Indigenous populations of Australia (Australian Bureau of Statistics 2004). The NLES asks respondents have any of these things [list of 'stressors' or 'negative life events'] been a problem for you or your family or friends during the last year? Respondents then answer 'yes' or 'no' to a list of 12 'stressors' or 'negative life events', which are: gambling problem; divorce or separation; death of family member or close friend; serious illness or disability; serious accident; alcohol or drug related problems; not able to get a job; lost job, made redundant, sacked; witness to violence; victim of abuse or violent crime; trouble with the police; and mental illness.

It is clear from the wording of the NLES question that the instrument does not measure problem gambling prevalence or prevalence for any of the items. It asks respondents if gambling has ... been a problem for you, your family or close friends during the last year. Therefore, the NLES gambling problem item measures the reach or extent of gambling problems throughout peoples' social and family networks and is not an individual measure of problem gambling. This broader conceptualisation of gambling-related harm is consistent with the Australian definition of problem gambling which states that "problem gambling is characterised by difficulties in limiting money and/or time spent on gambling which leads to adverse consequences for the gambler, others, or for the communities" (Neal et al. 2005, p 126).

\section{Explanatory variables}

There is no single variable contained in the GSS indicating a respondents' CALD status and therefore it had to be derived from related variables. Two variables, region of birth and main language spoken at home were used to generate a CALD status variable that indicated whether the respondent was both born overseas and did not speak English at home. Other variables related to CALD status were main language spoken at home, level in spoken English, region of birth, and year of arrival in Australia. The GSS also contains a large number of variables from demographic (age sex, location, marital status, crowding, household and family type), socioeconomic status (tenure type, income, education), financial stress (ran out of money, raise money in emergency), social connectedness (participation in a range of social and sporting activities and events) and health (self-reported health) related domains that are used in the analyses.

\section{Statistical analysis}

The distribution of CALD related social connectedness variables, and estimates of NLES items are given for the 2002 and 2006 surveys. Statistical differences were determined by dividing the absolute difference between the estimates by the standard error of the 
difference $\left(\sqrt{S E_{1}^{2}+S E_{2}^{2}}\right)$, where $S E_{1}$ and $S E_{2}$ are the standard errors of the estimates being compared respectively, to obtain a test statistic for comparison with Student's t-distribution. Statistical differences were determined for CALD related variables between the 2002 and 2006 CALD populations; for social connectedness variables between the 2006 CALD and non-CALD populations; and for NLES items between 2002 and 2006 CALD populations, and between CALD and non-CALD populations for 2002 and 2006.

The outcome variable, reported gambling problem is dichotomous and therefore well suited to logistic regression modelling. Separate models for the CALD and non-CALD populations were unable to be generated due to the small percentage of the CALD population reporting gambling problems and the relatively small size of this subpopulation group. Therefore models for the total population were first generated and then CALD related variables were substituted into final models to assess if the CALD status or any related variables showed an independent association with reported gambling problems after controlling for other variables.

First, unadjusted associations between reported gambling problem and all explanatory variables, including those relating to CALD status (region of birth, year of arrival, language region, proficiency in English and the CALD status variable) were assessed. Explanatory variables (but not CALD related variables) showing a significant $(\mathrm{p} \leq 0.05)$ association with gambling problems were then assessed for collinearity to ensure the assumptions associated with logistic regression modelling were adhered to. Where two or more explanatory variables were significantly correlated, these were first entered into a separate model and variable(s) remaining significant were retained for the next stage. Next, significant explanatory variables were entered simultaneously into a multivariable logistic regression model and backward elimination carried out with removal of variables set at $\mathrm{p}>0.05$. Lastly, CALD-related variables were added to models to determine if any had an adjustyed association with reported gambling problems.

All analyses were carried out using Stata v9.2 accessed via the ABS Remote Access Data Laboratory or RADL (Australian Bureau of Statistics 2006) using the expanded confidentialised unit record files (CURF) for the 2002 and 2006 GSS's. Data was weighted to the Estimated Resident Population for non-remote Australia at the time of the survey, and survey replicate method (SVR) set of commands was used to analyse data (Winter 2008). All confidence intervals were calculated using the Jack Knife (jk1) method and adjusted for the survey design.

\section{Results}

Table 1 shows the distribution of CALD related variables for 2002 and 2006 . Statistically significant differences were present for main language spoken at home (South European, East European, East Asian) and region of birth (Europe, North/ South/East Asia) and year of arrival (Recent, Long term). In 2002, 13\% of Australia's adult population were defined as CALD, increasing to $13.3 \%$ in 2006.

Table 2 reports estimates for variables related to social connectedness for the 2006 CALD and non-CALD populations, and the 2002 CALD population. All measures of social connectedness, except participation in church or religious activities, indicated lower levels of participation for the CALD population in 2006. Social support amongst the CALD population was also significantly lower than in the non-CALD population. 
Table 1 Distribution of CALD status for the total Australian population and the distribution of CALD-related variables for the CALD population, 2002 and 2006

\begin{tabular}{|c|c|c|c|c|}
\hline \multirow[t]{2}{*}{ Culture and language variables } & 2002 & 2006 & t-value & \multirow[t]{2}{*}{ p-value } \\
\hline & $\%(\mathrm{SE})$ & $\%$ (SE) & & \\
\hline \multicolumn{5}{|l|}{ Total Australian population } \\
\hline \multicolumn{5}{|l|}{ CALD: Birthplace and language groups } \\
\hline \multicolumn{5}{|l|}{ Non-CALD population } \\
\hline Australia \& English at home & $69.4(0.5)$ & $68.7(0.7)$ & 0.81 & 0.416 \\
\hline Australia \& not English at home & $3.0(0.2)$ & $3.1(0.3)$ & 0.28 & 0.782 \\
\hline Overseas \& English at home & $14.6(0.4)$ & $14.8(0.3)$ & 0.40 & 0.689 \\
\hline \multicolumn{5}{|l|}{ CALD population } \\
\hline Overseas \& not English at home & $13.0(0.4)$ & $13.3(0.5)$ & 0.47 & 0.639 \\
\hline \multicolumn{5}{|l|}{ CALD status } \\
\hline Non-CALD population & $87.0(0.4)$ & $86.7(0.5)$ & 0.47 & 0.639 \\
\hline CALD population & $13.0(0.4)$ & $13.3(0.5)$ & 0.47 & 0.639 \\
\hline Total Australian population & 100.0 & 100.0 & - & - \\
\hline N (weighted population) & $14,548,869$ & $15,307,066$ & - & - \\
\hline \multicolumn{5}{|l|}{ Total CALD population } \\
\hline \multicolumn{5}{|l|}{ Main language spoken at home } \\
\hline North European & $4.8(0.7)$ & $6.3(0.7)$ & 1.52 & 0.130 \\
\hline South European & $25.6(1.6)$ & $19.3(1.6)$ & 2.78 & 0.005 \\
\hline East European & $15.9(1.0)$ & $11.0(1.4)$ & 2.85 & 0.004 \\
\hline SW Asian central & $9.4(1.0)$ & $10.3(1.4)$ & 0.52 & 0.601 \\
\hline South Asian & $9.2(0.9)$ & $9.9(1.0)$ & 0.52 & 0.603 \\
\hline SE Asian & $11.9(1.2)$ & $14.9(1.8)$ & 1.39 & 0.166 \\
\hline East Asian & $18.5(1.3)$ & $23.3(1.8)$ & 2.16 & 0.031 \\
\hline Other language & $4.6(0.7)$ & $4.9(0.9)$ & 0.26 & 0.792 \\
\hline \multicolumn{5}{|l|}{ Level in spoken English } \\
\hline Very well & $35.9(1.6)$ & $38.4(2.0)$ & 0.98 & 0.329 \\
\hline Well & $38.7(1.5)$ & $38.0(2.1)$ & 0.27 & 0.786 \\
\hline Not well & $23.0(1.7)$ & $20.8(1.3)$ & 1.03 & 0.304 \\
\hline None & $2.4(0.5)$ & $2.8(0.6)$ & 0.51 & 0.609 \\
\hline \multicolumn{5}{|l|}{ Region of birth } \\
\hline Europe & $40.8(1.8)$ & $32.4(2.3)$ & 2.88 & 0.004 \\
\hline Africa/Middle East & $11.5(0.9)$ & $14.1(1.5)$ & 1.49 & 0.137 \\
\hline North/South/East Asia & $30.9(1.5)$ & $38.1(2.1)$ & 2.79 & 0.005 \\
\hline India/Central Asia & $7.8(0.9)$ & $8.7(1.1)$ & 0.63 & 0.527 \\
\hline New Zealand/Oceania & $4.5(0.8)$ & $3.7(0.8)$ & 0.71 & 0.480 \\
\hline Americas and not stated & $4.6(0.7)$ & $2.9(0.7)$ & 1.72 & 0.086 \\
\hline \multicolumn{5}{|l|}{ Year of arrival } \\
\hline $2001-2006 / 2002$ (recent) $^{1}$ & $5.1(0.8)$ & $16.9(1.6)$ & 6.60 & 0.000 \\
\hline 1991-2000 (medium) & $26.8(1.8)$ & $23.0(1.7)$ & 1.53 & 0.125 \\
\hline Before 1991 (long term) & $68.1(1.9)$ & $60.2(1.8)$ & 3.02 & 0.003 \\
\hline Total CALD population & 100.0 & 100.0 & - & - \\
\hline N (weighted population) & $1,891,353$ & $2,034,595$ & - & - \\
\hline
\end{tabular}

NOTES: Percentages may not add to $100 \%$ due to rounding.

Totals for 18 years and over.

1 Recent $=2001-2002$ in the 2002 GSS and Recent $=2001-2006$ in the 2006 GSS . 
Table 2 Distribution of social connectedness variables by CALD status: 2006 GSS

\begin{tabular}{|c|c|c|c|c|c|}
\hline \multirow{3}{*}{$\begin{array}{l}\text { Social connectedness and } \\
\text { support variables }\end{array}$} & 2006 & 2006 & t-value & \multirow[t]{3}{*}{ p-value } & \multirow{3}{*}{$\begin{array}{r}2002 \\
\text { CALD } \\
\% \text { (SE) }\end{array}$} \\
\hline & CALD & Non-CALD & & & \\
\hline & $\%$ (SE) & $\%$ (SE) & & & \\
\hline \multicolumn{6}{|l|}{ Social activities last 12 months } \\
\hline \multicolumn{6}{|c|}{ Adult education/special interest group } \\
\hline None & $42.5(1.6)$ & $32.0(0.6)$ & 6.14 & 0.000 & nc \\
\hline Participated in & $57.5(1.6)$ & $68.0(0.6)$ & 6.14 & 0.000 & nc \\
\hline \multicolumn{6}{|l|}{ Church or religious } \\
\hline None & $69.2(1.7)$ & $82.0(0.5)$ & 7.22 & 0.000 & $60.4(1.5)$ \\
\hline Participated in religion & $30.8(1.7)$ & $18.0(0.5)$ & 7.22 & 0.000 & $39.6(1.5)$ \\
\hline \multicolumn{6}{|l|}{ Restaurant/cafe/bar/social club } \\
\hline None & $88.3(1.4)$ & $78.7(0.5)$ & 6.46 & 0.000 & nc \\
\hline Attended & $11.7(1.4)$ & $21.3(0.5)$ & 6.46 & 0.000 & nc \\
\hline \multicolumn{6}{|l|}{ Sports/physical activity } \\
\hline None & $81.3(1.7)$ & $63.4(0.6)$ & 9.93 & 0.000 & $67.4(1.9)$ \\
\hline Participate/attended/watched & $18.7(1.7)$ & $36.6(0.6)$ & 9.93 & 0.000 & $32.6(1.9)$ \\
\hline \multicolumn{6}{|l|}{ Arts/craft group } \\
\hline None & $93.4(0.9)$ & $83.7(0.4)$ & 9.85 & 0.000 & nc \\
\hline Visited & $6.6(0.9)$ & $16.3(0.4)$ & 9.85 & 0.000 & nc \\
\hline \multicolumn{6}{|l|}{ Leisure/culture/recreation } \\
\hline None & $18.7(1.3)$ & $10.3(0.3)$ & 6.30 & 0.000 & $20.2(1.2)$ \\
\hline Attended & $81.3(1.3)$ & $89.7(0.3)$ & 6.30 & 0.000 & $79.8(1.2)$ \\
\hline \multicolumn{6}{|l|}{ Sport/physical activity attendance } \\
\hline None & $73.3(2.1)$ & $44.0(0.6)$ & 13.42 & 0.000 & $74.3(1.5)$ \\
\hline Attended & $26.7(2.1)$ & $56.0(0.6)$ & 13.42 & 0.000 & $25.7(1.5)$ \\
\hline \multicolumn{6}{|l|}{ Sport/physical activity participation } \\
\hline None & $48.6(2.3)$ & $36.1(0.8)$ & 5.13 & 0.000 & $51.7(1.5)$ \\
\hline Participated & $51.4(2.3)$ & $63.9(0.8)$ & 5.13 & 0.000 & $48.3(1.5)$ \\
\hline \multicolumn{6}{|c|}{ Support if needed help in last 12 months } \\
\hline No support & $13.5(0.8)$ & $5.6(0.3)$ & 9.25 & 0.000 & $12.1(1.0)$ \\
\hline Support & $86.5(0.8)$ & $94.4(0.3)$ & 9.25 & 0.000 & $87.9(1.0)$ \\
\hline Total & 100.0 & 100.0 & & & 100.0 \\
\hline $\mathrm{N}$ (weighted population) & $2,034,595$ & $13,272,471$ & & & $1,891,353$ \\
\hline Australia & $13.3(0.5)$ & $86.7(0.5)$ & & & $13.0(0.4)$ \\
\hline
\end{tabular}

NOTES: Percentages may not add to $100 \%$ due to rounding.

$n c=$ Non-comparable due to different wording in questions between 2002 and 2006 surveys.

Figures 3 and 4 present estimates for NLES items by CALD status for the 2002 and 2006 populations' respectively. In 2002, there was no evidence of a difference between a report of 'gambling problem' in the CALD and non-CALD populations respectively (3.3\% and 3.5\%). In 2002, estimates for all NLES items were statistically lower in the CALD population compared with the non-CALD population, except for 'gambling problem,' 'not able to get a job (no job),' 'death of a family member', and 'other stressor'. The most commonly reported stressor for the CALD population was 'death of family member' $(17.5 \%)$, while for the non-CALD population it was having or living with someone with a 'chronic illness' (24.5\%). 


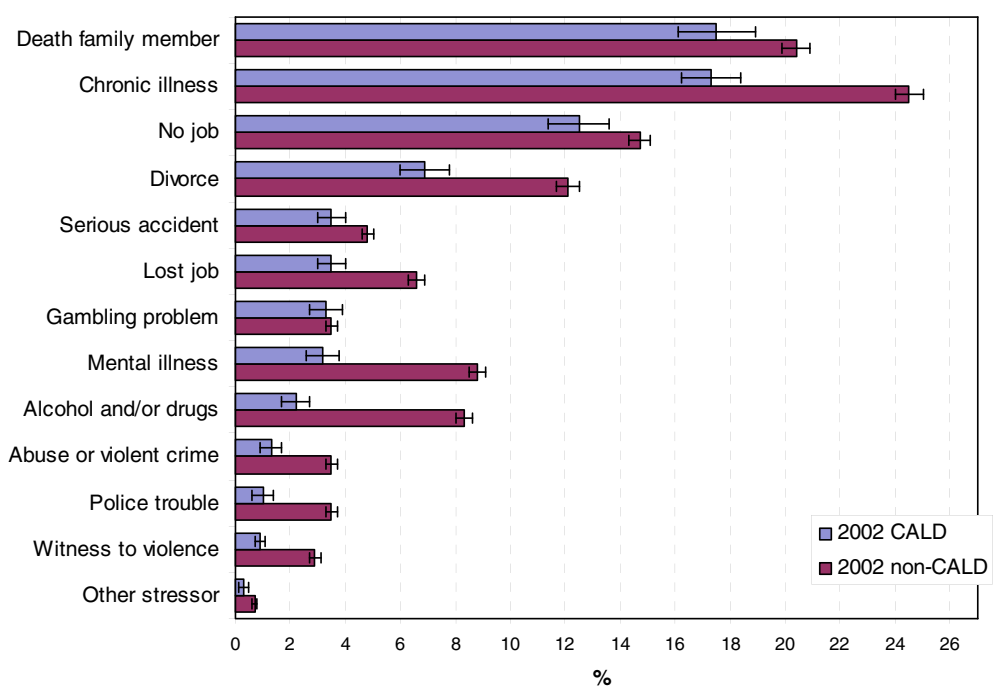

Figure 3 NLES item estimates (standard errors) by CALD status for 2002.

Estimates of NLES items in 2006 were all statistically lower for the CALD population except for 'not able to get a job' and 'other stressor'. Estimates for reported 'gambling problem' were $1.3 \%$ and $3.5 \%$ for the CALD and non-CALD 2006 populations respectively. Living with someone or having a 'chronic illness' was reported most frequently for the CALD (20.3\%) and non-CALD (20.7\%) 2006 populations. Comparing the 2002 and 2006 CALD populations, only one NLES item, 'gambling problem' showed evidence of a difference (3.3\% to $1.3 \%)$.

Table 3 presents unadjusted associations between CALD status and related variables and reported gambling problems. None of the CALD related variables had a significant unadjusted association with reported gambling problems in the 2002 survey. When the variables country of birth and main language spoken at home were combined, the category of being born overseas and speaking English at home, was marginally nonsignificant $(\mathrm{p}=0.080)$ and associated with reduced levels of reported gambling problems (Odds Ratio (OR) 0.69, 95\% confidence interval (CI) 0.51 to 0.93 ).

In 2006, the CALD status variable and a number of other CALD related variables showed a significant association with reported gambling problems. Being a member of the CALD population was significantly associated with reduced levels of reporting gambling problems (OR 0.38 , CI 0.21 to 0.68). The variable combining country of birth and main language spoken at home was also significantly associated with reported gambling problems, with people not born in Australia and not speaking English at home (equivalent to the CALD population) being associated with reduced reporting of gambling problems, while people born in Australia and not speaking English at home had marginally non-significant association with increased reporting of gambling problems (OR 2.14, CI 0.95 to 4.85). People who spoke either a southern, south-eastern or eastern Asian language reported fewer gambling problems (OR 0.14, CI 0.04 to 0.54), though this was marginally non-significant $(\mathrm{p}=0.067)$, while people not speaking English very well, also reported significantly $(\mathrm{p}=0.015)$ fewer gambling problems $(\mathrm{OR} 0.30, \mathrm{CI} 0.14$ to 0.65 ). People whose region of birth was from New Zealand or Oceania reported significantly higher levels of gambling problems (OR 2.16, CI 1.04 to 4.49). 


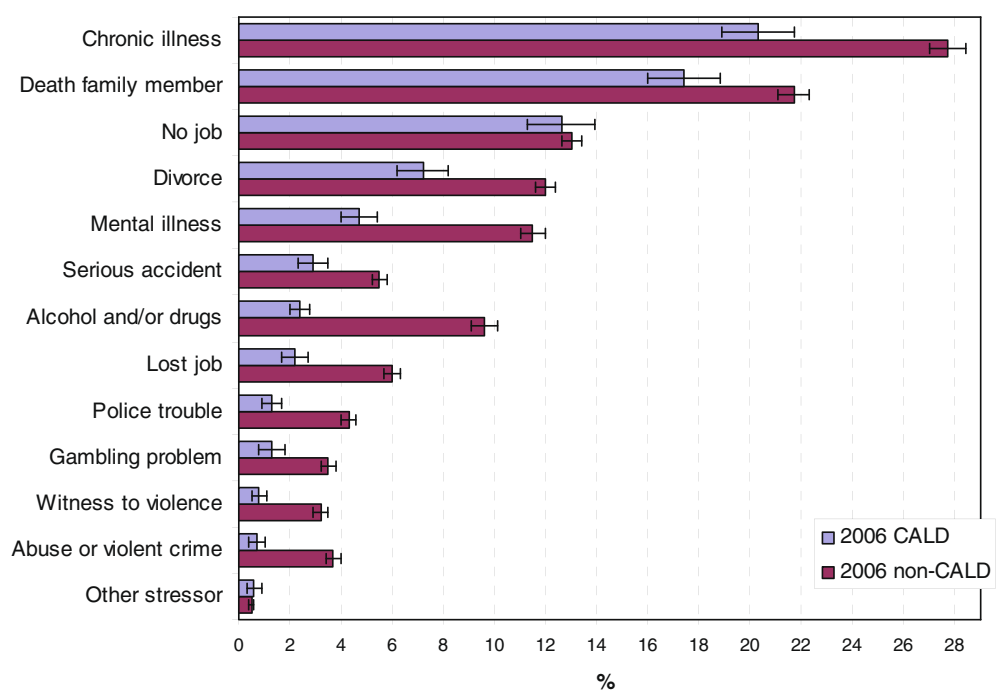

Figure 4 NLES item estimates (standard errors) by CALD status for 2006.

No models are presented for the 2002 survey as no CALD related variables showed a significant association with reported gambling problems, and multivariable adjusted models for reported gambling problems have been reported in previous work (Stevens and Young 2009; Stevens et al. 2010). Table 4 presents multivariable adjusted models for reported gambling problems from the 2006 survey. Two models are presented that contain variables related to CALD status which remained significant after adjusting for all other significant correlates of reported gambling problems. Model 1 includes the combination variable, country of birth and main language spoken at home, with people born overseas and not speaking English at home having reduced odds of reporting a gambling problem (OR 0.53, CI 0.30 to 0.95), after adjustment for state/territory, age, cash flow problems, social participation, victim of physical or threatened violence and self-assessed health.

In Model 2, the CALD status variable and the region of birth variable remained significant after adjustment for all other significant correlates of reported gambling problems (Table 2: Model 2). Being a member of the CALD population was associated with reduced odds of reporting a gambling problem (OR 0.47, CI 0.26 to 0.85 ), while being born in either New Zealand or Oceania was associated with increased odds (OR 2.13, CI 1.00 to 4.52). All other variables reported for Model 1 remained significant in this model with minimal change in reported odds ratios.

\section{Discussion}

\section{Using population surveys to measure gambling problems in the CALD population}

This paper presented an analysis of gambling problems in the CALD population using nationally representative ABS population surveys. The analyses, while providing an overall picture of reported gambling problems amongst individuals, their family and close friends for the CALD population of Australia, also raised a number of issues and questions regarding the utility of current ABS general social survey for this purpose. While gambling problem estimates for the total CALD population were able to be 
Table 3 Unadjusted associations between reported gambling problems and CALDrelated variables

\begin{tabular}{|c|c|c|c|}
\hline & 2002 & & 2006 \\
\hline OR $(95 \% \mathrm{Cl})$ & $\begin{array}{l}\text { Reported gambling } \\
\text { problems } \% \text { (SE) }\end{array}$ & OR $(95 \% \mathrm{Cl})$ & $\begin{array}{l}\text { Reported gambling } \\
\text { problems\% (SE) }\end{array}$ \\
\hline$(p=0.758) 1.00$ & $3.5(0.2)$ & $(p=0.002) 1.00$ & $3.5(0.3)$ \\
\hline $0.94(0.64-1.39)$ & $3.3(0.6)$ & $0.38(0.21-0.68)$ & $1.3(0$ \\
\hline
\end{tabular}

Country of birth and language

\begin{tabular}{|c|c|c|c|c|}
\hline $\begin{array}{l}\text { Australia and English } \\
\text { at home }\end{array}$ & $(p=0.080) 1.00$ & $3.6(0.2)$ & $(p=0.017) 1.00$ & $3.3(0.2)$ \\
\hline $\begin{array}{l}\text { Australia and not } \\
\text { English at home }\end{array}$ & $1.74(0.85-3.59)$ & $6.1(1.7)$ & $2.14(0.95-4.85)$ & $6.7(2.4)$ \\
\hline $\begin{array}{l}\text { Overseas \& English } \\
\text { at home }\end{array}$ & $0.69(0.51-0.93)$ & $2.5(0.4)$ & $1.13(0.71-1.80)$ & $3.7(0.7)$ \\
\hline $\begin{array}{l}\text { Overseas \& not } \\
\text { English at home }\end{array}$ & $0.92(0.61-1.38)$ & $3.3(0.6)$ & $0.40(0.23-0.72)$ & $1.3(0.4)$ \\
\hline \multicolumn{5}{|l|}{ Language region ${ }^{2}$} \\
\hline Australia & $(p=0.252) 1.00$ & $3.4(0.2)$ & $(p=0.067) 1.00$ & $3.3(0.2)$ \\
\hline North European & $0.52(0.01-41.2)$ & $1.8(1.6)$ & $1.72(0.37-7.97)$ & $5.6(3.2)$ \\
\hline South/East European & $1.39(0.73-2.64)$ & $4.6(1.2)$ & $0.94(0.44-1.97)$ & $3.1(1.0)$ \\
\hline South-west Asian & $2.41(1.10-5.27)$ & $7.8(2.6)$ & $0.65(0.17-2.45)$ & $2.2(1.2)$ \\
\hline $\begin{array}{l}\text { South/South-east/ } \\
\text { East Asian }\end{array}$ & $0.45(0.19-1.09)$ & $1.6(0.6)$ & $0.14(0.04-0.54)$ & $0.5(0.2)$ \\
\hline $\begin{array}{l}\text { Other language } \\
\text { region }\end{array}$ & $1.70(0.51-5.71)$ & $5.6(2.8)$ & $2.87(0.79-10.4)$ & $9.0(4.5)$ \\
\hline \multicolumn{5}{|l|}{ Proficiency in English } \\
\hline $\begin{array}{l}\text { Speaks English at } \\
\text { home }\end{array}$ & $(p=0.334) 1.00$ & $3.4(0.2)$ & $(p=0.015) 1.00$ & $3.3(0.2)$ \\
\hline Very well & $1.38(0.84-2.26)$ & $4.6(0.9)$ & $1.15(0.64-2.07)$ & $3.8(1.0)$ \\
\hline Well/not well/none & $0.92(0.58-1.46)$ & $3.1(0.7)$ & $0.30(0.14-0.65)$ & $1.0(0.4)$ \\
\hline \multicolumn{5}{|l|}{ Region of birth ${ }^{2}$} \\
\hline Australia & $(p=0.531) 1.00$ & $3.7(0.2)$ & $1.34(0.91-1.97)$ & $3.4(0.3)$ \\
\hline Europe & $0.73(0.45-1.18)$ & $2.7(0.6)$ & $0.71(0.48-1.04)$ & $2.4(0.4)$ \\
\hline Africa/Middle East & $0.95(0.44-2.07)$ & $3.5(1.3)$ & $0.84(0.36-1.99)$ & $2.7(1.1)$ \\
\hline $\begin{array}{l}\text { North/South/East } \\
\text { Asia }\end{array}$ & $0.53(0.22-1.28)$ & $2.0(0.8)$ & $0.61(0.23-1.60)$ & $2.0(0.8)$ \\
\hline $\begin{array}{l}\text { India/North/West } \\
\text { Asia }\end{array}$ & $0.49(0.04-5.69)$ & $1.9(1.4)$ & ne & $0.4(0.4)$ \\
\hline $\begin{array}{l}\text { New Zealand/ } \\
\text { Oceania }\end{array}$ & $1.35(0.73-2.50)$ & $4.9(1.3)$ & $2.16(1.04-4.49)$ & $6.4(2.0)$ \\
\hline Americas/not stated & $0.85(0.18-3.98)$ & $3.2(1.9)$ & $0.29(0.02-4.15)$ & $1.0(0.7)$ \\
\hline \multicolumn{5}{|l|}{ Time in Australia } \\
\hline Born in Australia & $(p=0.354) 1.00$ & $3.7(0.2)$ & $(p=0.266) 1.00$ & $3.4(0.3)$ \\
\hline $\begin{array}{l}\text { Recent } \\
(2001-2002 / 2006)\end{array}$ & $0.64(0.10-3.95)$ & $2.4(1.6)$ & $0.66(0.22-2.02)$ & $2.3(1.1)$ \\
\hline
\end{tabular}


Table 3 Unadjusted associations between reported gambling problems and CALDrelated variables (Continued)

\begin{tabular}{|c|c|c|c|c|}
\hline $\begin{array}{l}\text { Medium } \\
(1991-2000)\end{array}$ & $0.61(0.29-1.27)$ & $2.3(0.7)$ & $1.22(0.67-2.22)$ & $4.1(1.1)$ \\
\hline $\begin{array}{l}\text { Long-term } \\
\text { (before 1991) }\end{array}$ & $0.82(0.58-1.16)$ & $3.0(0.5)$ & $0.65(0.41-1.03)$ & $2.2(0.4)$ \\
\hline
\end{tabular}

NOTES: Bold font indicates statistically significant difference $(p \leq 0.05)$.

1 This category is the same as the CALD group in the CALD status variable.

2 For 2006 data, the small sample size restricted the bivariate analysis, so odds ratios for Region of Birth are calculated

for individual regions, with the reference category being all other regions including Australia.

$n e=$ Not estimable due to small sample size for this group.

produced, estimates for states and territories could not be produced with adequate accuracy. Further, the GSS is a population level survey, and CALD sub-populations may not be randomly sampled within the overall sample, due to the patchy nature of their distribution across Australia (Markus, et al. 2009). The small sample sizes associated with sub-populations within the CALD population also gave rise to unacceptably high standard errors associated with estimates of reported gambling problems for CALD sub-populations. This also limited the power of the statistical models to detect evidence that gambling problems were occurring at significantly higher (or lower) levels than in the non-CALD population.

In addition to these survey and statistical problems, the composition of CALD population is not stable over time due to the changing circumstances in which people immigrate to Australia. There are three primary reasons for this:

1) Australia has substantially increased its skilled immigrant intake to lessen the effects of the skills shortage in the job market;

2) the stability of various countries around the world from which migrants move to Australia is more often than not in a state of flux, changes substantially over time and is dependent on circumstances outside of the control of Australian immigration policy; and 3) the heterogeneity within the CALD population, which includes a diverse range of different cultural attributes (e.g. collectivist $c f$. individualistic cultures).

Related to this third point, the definition used to identify the CALD population excludes approximately $18 \%$ of the adult population that could also be considered part of the CALD population (see 'country of birth' and 'language spoken at home' variable in Table 1). For example, 3\% of the adult population were born in Australia and do not speak English at home - these people were not included in the CALD population derived from the survey data used in the analyses. Furthermore, some 13\% of adults were born overseas and spoke English at home, of which an unknown percentage would be multi-lingual and would very likely exhibit characteristics of the CALD population as defined for the analyses.

The CALD population is not a homogenous population group either, so from a statistical viewpoint it is not a clearly identifiable population. Within the CALD population there will be people of different religions, from different countries and from differing circumstances (e.g. skilled migrants, refugees from war torn countries). These factors lead to the CALD population, as a grouped entity being heterogeneous, which means that issues that may be occurring for various segments within this population may remain obscured. 
Table 4 Multivariable models for 2006 reported gambling problems

\begin{tabular}{|c|c|c|c|}
\hline Explanatory variables & $\begin{array}{r}\text { Model } 1 \\
\text { OR }(95 \% \mathrm{Cl})\end{array}$ & $\begin{array}{r}\text { Model } 2 \\
\text { OR }(95 \% \mathrm{Cl})\end{array}$ & $\begin{array}{r}\text { Reported } \\
\text { gambling } \\
\text { problems } \\
\%(\mathrm{SE})\end{array}$ \\
\hline \multicolumn{4}{|l|}{ CALD status } \\
\hline Non-CALD & na & $(p=0.015) 1.00$ & $3.5(0.3)$ \\
\hline CALD & na & $0.47(0.26-0.85)$ & $1.3(0.4)$ \\
\hline \multicolumn{4}{|l|}{ Country of birth and language } \\
\hline Australia and English at home & $(p=0.064) 1.00$ & na & $3.3(0.2)$ \\
\hline Australia and not English at home & $1.58(0.64-3.87)$ & na & $6.7(2.4)$ \\
\hline Overseas and English at home & $1.48(0.92-2.37)$ & na & $3.7(0.7)$ \\
\hline Overseas and not English at home & $0.53(0.30-0.95)$ & na & $1.3(0.4)$ \\
\hline \multicolumn{4}{|l|}{ Region of birth } \\
\hline Any other country (incl. Australia) & na & $(p=0.049) 1.00$ & $3.1(0.2)$ \\
\hline New Zealand/Oceania & na & $2.13(1.00-4.52)$ & $6.4(2.0)$ \\
\hline \multicolumn{4}{|l|}{ Proficiency in English language } \\
\hline Speaks at home & & dropped & $3.3(0.2)$ \\
\hline Very well & & dropped & $3.8(1.0)$ \\
\hline Well/not well/none & & dropped & $1.0(0.4)$ \\
\hline \multicolumn{4}{|l|}{ State/Territory } \\
\hline WA & 1.00 & 1.00 & $1.5(0.4)$ \\
\hline NSW & $2.58(1.35-4.92)$ & $2.39(1.27-4.51)$ & $3.3(0.5)$ \\
\hline VIC & $2.60(1.44-4.69)$ & $2.45(1.34-4.47)$ & $3.2(0.5)$ \\
\hline QLD & $2.45(1.41-4.23)$ & $2.31(1.32-4.02)$ & $3.6(0.5)$ \\
\hline SA & $2.70(1.54-4.73)$ & $2.66(1.52-4.67)$ & $3.6(0.5)$ \\
\hline NT & $2.65(1.61-4.36)$ & $2.57(1.57-4.22)$ & $4.5(0.6)$ \\
\hline $\mathrm{ACT}$ & $3.26(1.79-5.92)$ & $3.14(1.73-5.69)$ & $4.3(0.5)$ \\
\hline TAS & $1.93(1.01-3.71)$ & $1.87(0.96-3.67)$ & $2.4(0.4)$ \\
\hline \multicolumn{4}{|l|}{ Age (years) } \\
\hline $18-24$ & $1.90(1.12-3.21)$ & $1.84(1.12-3.05)$ & $4.0(0.9)$ \\
\hline $25-34$ & $2.83(1.68-4.75)$ & $2.71(1.66-4.45)$ & $4.3(0.6)$ \\
\hline $35-44$ & $2.70(1.61-4.53)$ & $2.64(1.62-4.30)$ & $4.0(0.4)$ \\
\hline $45-54$ & $2.59(1.41-4.73)$ & $2.54(1.40-4.63)$ & $3.7(0.7)$ \\
\hline 55 or more & 1.00 & 1.00 & $1.3(0.2)$ \\
\hline \multicolumn{4}{|l|}{ Cash flow problems } \\
\hline No cash flow problems & 1.00 & 1.00 & $2.3(0.2)$ \\
\hline One problem & $1.62(0.97-2.70)$ & $1.63(0.98-2.72)$ & $4.8(1.0)$ \\
\hline Two or more problems & $2.39(1.61-3.55)$ & $2.40(1.61-3.59)$ & $8.6(1.0)$ \\
\hline \multicolumn{4}{|l|}{ Social participation last 12 months } \\
\hline Sport and recreation & $1.36(1.05-1.76)$ & $1.40(1.08-1.80)$ & $4.0(0.4)$ \\
\hline No sport and recreation & 1.00 & 1.00 & $2.7(0.2)$ \\
\hline \multicolumn{4}{|l|}{ Social participation last 12 months } \\
\hline Arts and crafts & $1.53(1.03-2.27)$ & $1.55(1.04-2.31)$ & $4.5(0.7)$ \\
\hline No arts and crafts & 1.00 & 1.00 & $2.9(0.3)$ \\
\hline \multicolumn{4}{|l|}{ Physical or threatened violence } \\
\hline Victim & $2.94(2.16-4.01)$ & $2.99(2.18-4.10)$ & $9.8(1.0)$ \\
\hline
\end{tabular}


Table 4 Multivariable models for $\mathbf{2 0 0 6}$ reported gambling problems (Continued)

\begin{tabular}{lrrr}
\hline Not a victim & 1.00 & 1.00 & $2.4(0.2)$ \\
Self assessed health & & & \\
Excellent & 1.00 & 1.00 & $2.5(0.5)$ \\
Very good & $1.13(0.68-1.88)$ & $1.11(0.67-1.84)$ & $2.7(0.3)$ \\
Good & $\mathbf{1 . 6 6 ( \mathbf { 1 . 0 4 } - \mathbf { 2 . 6 3 } )}$ & $\mathbf{1 . 6 2 ( 1 . 0 2 - 2 . 5 8 )}$ & $4.0(0.5)$ \\
Fair & $1.75(0.90-3.40)$ & $1.71(0.89-3.29)$ & $3.5(0.8)$ \\
Poor & $1.96(0.93-4.15)$ & $1.92(0.92-4.02)$ & $4.1(1.1)$ \\
\hline NOTE: Bold font indicates statistically significant difference $(\mathrm{p} \leq 0.05)$. & &
\end{tabular}

In terms of data quality and quantity, the GSSs have limited information on gambling and associated concepts (e.g. belief in luck, frequency of gambling, game preferences, and time and money spent gambling). Therefore, the analyses could not relate the measurement of gambling problems to the gambling habits and potential problems associated with gambling by members of the CALD population (or the non-CALD population). However, the strong associations with social connectedness variables did suggest that the CALD population, as a whole, were less socially connected and experienced fewer life stressors, including gambling problems, than the non-CALD population.

The subjective nature of the module that captured information on gambling problems could also be influencing findings for the CALD population, as this group may be less reluctant to report problems, even if they do exist (Cultural Perspectives Pty Ltd 2005). There is no way of testing this possibility, though the ABS goes through extensive testing for all survey questions used in the General Social Survey (Australian Bureau of Statistics 2003, 2007). The following section now discusses what could be ascertained from the analyses of gambling problems amongst the CALD population. However, the previously noted caveats should be considered in the following interpretations.

\section{The relationship between CALD status and reported gambling problems}

In the analysis of the 2002 survey, there was no evidence of an association between gambling problems and being a member of the CALD population (or related variables). However, the 2006 analysis revealed that being a member of the CALD population was protective of gambling problems and this association remained after controlling for other variables that showed evidence of an association with reported gambling problems (State/Territory, age, financial stress, social connectedness, being a victim of physical or threatened violence, and self-assessed health).

Social connectedness has been shown to be protective against developing problems associated with gambling in some studies (Escobar, et al. 2000; Tanasornnarong, et al. 2004). However, the 2006 multivariable model for the total population showed that people who were more likely to be socially connected were more likely to report a gambling problem for themselves or someone in the family and social networks. Importantly, social connectedness variables were lower amongst the CALD population (except for attending religious activities), as were most estimates of stressors collected as part 
of the NLES. These two findings alone support the notion that the CALD population as a whole, experience fewer gambling-related problems.

Problems with acculturation for immigrants have also been shown to be associated with problem gambling in CALD communities (Brozovic-Basic 2005; Scull and Woolcock 2005), though in the current analysis there was no evidence of an association between year of arrival and reported gambling problems. The lack of an association between this year of arrival and reported gambling problems is most likely attributable to heterogeneity within the CALD population and the smaller sample size when crosstabulating variables.

Another possibility explaining the lower levels of gambling problems observed for the CALD population may relate to actually not being able to speak English and therefore not attending places where gambling facilities are available. In all variables relating to social connectedness except for attending church or religious activities (which is protective of problem gambling), the CALD population had lower participation. So, if the CALD population does not socialise in places where commercial gambling is available, then language is acting as a barrier to access, therefore limiting their interaction with gambling activities such as electronic gambling machines (EGMs or 'pokies'). This may be important, as EGMs are the most common form of gambling available in places where people socialise in Australia, and is also the most risky form of gambling, in terms of developing gambling-related problems (Productivity Commission 2010).

Given the change in migrant intake over the last decade or two, the lower levels of gambling problems and social connectedness, may be a result of migrants not having had time to be cultured into EGM gambling and various Australian social activities. Additionally, many recent immigrants have been encouraged to live in regional locations (Department of Immigration and Citizenship 2009), and it may be that accessibility to gambling opportunities is limited (physically and socially), leading to lower levels of participation in gambling. Furthermore, many skilled immigrants come from middle-eastern countries where Islam is the predominant religion and gambling is specifically forbidden in the Koran (Binde 2005). Overall, this could translate into a lower percentage of this group developing problems associated with gambling, and lower participation in gambling leads to lower average time and money spent gambling, which in turn leads to lower levels of problem gambling. This is known as the consumption model, where the average amount consumed of a product increases with the percentage of the population that use the product and has been found to hold with alcohol and gambling (Lund 2006).

The literature reviewed indicates that some CALD sub-populations may have significant problems with gambling, but they are likely to be in the minority in their respective communities (Clarke et al. 2006; Cultural Partners Australia 2000; Loo, et al. 2008). Additionally, gambling participation rates have also been found to be lower amongst some CALD sub-populations compared with the general community (Cultural Partners Australia 2000). Only one sub-population of immigrants to Australia showed a significant association with reported gambling problem, those originating from New Zealand or Oceania. This finding is consistent with research in New Zealand, where Pacific Islanders and Maori populations have been found to have levels of problem gambling up to four times higher than the general New Zealand population after accounting for age differences (Abbott et al. 2004; Clarke, et al. 2006; Ministry of Health 2009). This 
finding may indicate the need to develop programs and improve services targeting this population group.

In the current study, the CALD population were more likely to be unemployed and have lower incomes, which are both risk factors associated with problem gambling (Hraba and Lee 1995; Shepherd et al. 1998; Young et al. 2008). However, the better levels of education observed for the CALD population would likely act as a protective factor with regards to developing gambling problems (Productivity Commission 1999, 2010), and are a by-product of Australia's recent migration policies that select for skilled immigrants (Department of Immigration and Citizenship 2009).

An important point is that the definition of the CALD population used for the current analyses excluded people born in Australia, who did not speak English at home. There was some evidence to suggest that this group experienced higher levels of gambling problems for themselves, family or friends. The combination variable of birth country and language spoken at home showed this group to have higher levels of reported gambling problems in both 2002 (6.1\%) and 2006 (6.7\%) than the non-CALD population (3.5\% and $3.5 \%$ respectively), though these elevated levels were marginally non-significant. This group represents a small percentage of the Australian adult population, which would partly contribute to the non-significance of the association between reported gambling problems and being a member of the CALD population. However, this highlights the subjective nature of 'CALD' as a label for use in research and policy. For example, if this group of non-English speaking Australians were included in the CALD population used in this analysis, it is possible that the finding that being in the CALD population is protective may not have been observed. More nuanced studies of ethnic groups within the CALD population are required to gain a better understanding of which groups are more at risk of developing gambling problems.

\section{Conclusions}

The analysis of gambling problems amongst the CALD population did not support the notion that gambling problems are occurring at higher levels amongst this group (as a whole) compared with the non-CALD population in Australia. Consistent with less reporting of gambling problems, the CALD population had lower reports of other life stressors measured in the NLES. There was also no evidence to suggest that immigrants who had settled in Australia at different times were more likely to report gambling problems. Consistent with the conceptual framework presented earlier, variables relating to a person's age, socioeconomic status, and social connectedness were all related to reports of gambling problems.

Future studies on Australia's CALD populations will require more targeted approaches. Perhaps a more effective future strategy is to carefully measure the characteristics of different immigration waves and/or populations to help identify their relationships to different forms of gambling behaviour. These characteristics may include, for example, not just 'country of origin' and 'language spoken at home', but exposure to violence and trauma, social position in their country of origin, English proficiency and skills/qualifications, refugee status, forms of economic participation in Australia and level of social integration as well as the usual battery of demographics measures. First, the specificity of these and other measures are more likely to help 
identify both risk and protective factors for gambling-related harm and the various ways that such groups gamble. Second, they may indicate where, when, to what purpose and for what motivation these groups gamble. What is required is more targeted research identifying which CALD populations are more vulnerable to problem gambling and more generally, identify the risk factors that may predispose people regardless of their cultural background. Another avenue may involve identifying people (and CALD sub-populations) within venues, rather than using population based surveys to monitor trends at a coarse level for CALD population.

\section{Key messages}

- The 2006 CALD population, as whole, experienced fewer gambling problems than the non-CALD population of Australia.

- The CALD population is a diverse population group that includes people who differ across important, demographic, socioeconomic, cultural and historical factors.

- National social surveys were limited in their usefulness for monitoring trends in gambling amongst the CALD population and sub-populations in Australia.

- More nuanced and targeted research designs are required to identify how gambling affects different CALD sub-populations.

Competing interests

The authors declare that they have no competing interests.

\section{Authors' contributions}

KG carried out the literature review that informed the introduction and discussion, and was partially responsible for drafting the introduction. MS carried out all statistical analyses and was responsible for the overall drafting of the manuscript, including introduction, methods, results and discussion. Both authors read and approved the final manuscript.

\section{Acknowledgements}

We would like to acknowledge two anonymous reviewers for comments on an earlier draft of this paper. We would also like to thank the Australian Bureau of Statistics for making their survey data available and the respondents who participated in the surveys.

Funding

Research grant CD/09/148478 from Gambling Research Australia made on behalf of the Ministerial Council on Gambling

Received: 2 February 2013 Accepted: 2 February 2013

Published: 20 February 2013

\section{References}

Abbott, MW, Volberg, RA, \& Rönnberg, S (2004). Comparing the New Zealand and Swedish National Surveys of Gambling and Problem Gambling. Journal of Gambling Studies, 20(3), 237-258.

Australian Bureau of Statistics (2003). General Social Survey, 2002: Data Reference package Cat. no. 4717.55.001. Canberra: ABS.

Australian Bureau of Statistics (2004). National Aboriginal and Torres Strait Islander Social Survey, Australia, 2002 (Cat. no. 4717.0.55.001). Canberra: ABS.

Australian Bureau of Statistics (2006). Remote Access Data Laboratory (RADL) User Guide. Version 4 (Cat. no. 1406.0.55.002). Canberra: ABS.

Australian Bureau of Statistics (2007). General Social Survey, 2006: Users Guide (Cat. no. 4159.55.002). Canberra: ABS.

Australian Bureau of Statistics (2008). Year Book, 2008 (Cat. no. 1301.0). Canberra: ABS.

Bandura, A (1986). Social foundations of thought and action. Englewood Cliffs, NJ: Prentice Hall.

Beattie, L, Blaszczynski, A, Maccallum, F, \& Joukhador, J (1999). Gambling problems in a multicultural society. Developing strategic alliances. Gold Coast: Paper presented at the Proceedings of the 9th National Association of Gambling Studies.

Binde, P (2005). Gambling Across Cultures: Mapping Worldwide Occurrence and Learning from Ethnographic Comparison. International Gambling Studies, 5(1), 1-27.

Breen, H, Hing, N, \& Gordon, A (2010). Exploring Indigenous Gambling: Understanding Indigenous Gambling Behaviour, Consequences, Risk Factors and Potential Interventions (pp. 1-242). Lismore: Southern Cross University.

Brozovic-Basic, K (2005). Pokie-free activities and places for Culturally and Linguistically Diverse Communities in Darebin Project. Darebin: Darebin City Council. 
Chantal, Y, \& Vallerand, RJ (1996). Skill versus luck: A motivational analysis of gambling involvement. Journal of Gambling Studies, 12(4), 407-418.

Chui, WH, \& O'Connor, I (2006). Understanding problem gambling in two ethnic communities in Brisbane, Queensland - A pilot study. Asia Pacific Journal of Social Work \& Development, 6(1), 67-75.

Clarke, D, Abbott, M, Tse, S, Townsend, S, Kingi, P, \& Manaia, W (2006). Gender, age, ethnic and occupational associations with pathological gambling in a New Zealand urban sample. New Zealand Journal of Psychology, 35(2), 84-91.

Clarke, D, Tse, S, Abbott, MW, Townsend, S, Kingi, P, \& Manaia, W (2007). Reasons for Starting and Continuing Gambling in a Mixed Ethnic Community Sample of Pathological and Non-problem Gamblers. International Gambling Studies, $7(3), 299-313$

Australia, CP (2000). The impact of gaming on specific cultural groups. Melbourne: Project report prepared for Victorian Casino and Gaming Authority.

Cultural Perspectives Pty Ltd (2005). Research into health promotion and best practice services for culturally and linguistically diverse communities. V. G. D. o. Melbourne, Victoria, Australia: Justice, Office for Gaming and Racing.

De La Rosa, M, Vega, R, \& Radisch, MA (2000). The Role of Acculturation in the Substance Abuse Behavior of AfricanAmerican and Latino Adolescents: Advances, Issues, and Recommendations. Journal of Psychoactive Drugs, 32(1), 33-42.

Department of Immigration and Citizenship (2009). Population flows: Immigration aspects 2007-08 edition. Canberra: Commonwealth of Australia.

Escobar, Jl, Nervi, CH, \& Gara, MA (2000). Immigration and Mental Health: Mexican Americans in the United States. Harvard Review of Psychiatry, 8(2), 64-72.

Gibbs Van Brunschot, E (2000). Gambling in context: The socio-cultural domain literature overview and annotated bibliography. Edmonton: Alberta Gaming Institute.

Hing, N, \& Breen, H (2001). Profiling Lady Luck: An Empirical Study of Gambling and Problem Gambling Amongst Female Club Members. Journal of Gambling Studies, 17(1), 47-69.

Hing, N, \& Haw, J (2009). The Development of a Multi-dimensional Gambling Accessibility Scale. Journal of Gambling Studies, 25(4), 569-581.

Hraba, J, \& Lee, G (1995). Problem gambling and policy advice: The mutability and relative effects of structural, associational and attitudinal variables. Journal of Gambling Studies, 11(2), 105-121.

Kidman, R (2004). Social networks: Good, bad or ugly? Weekly Addiction Gambling Education Report, 9(49), 8.

Ladouceur, R., \& Mireault, C. (1988). Gambling behaviors among high school students in the Quebec area. Journal of Gambling Studies, 4(1), 3-12.

Lesieur, HR, Cross, J, Frank, M, Welch, M, White, CM, Rubenstein, G, et al. (1991). Gambling and pathological gambling among university students. Addictive Behaviors, 16(6), 517-527.

Lesieur, HR, \& Heineman, M (1988). Pathological Gambling Among Youthful Multiple Substance Abusers in a Therapeutic Community. British Journal of Addiction, 83(7), 765-771.

Loo, J, Raylu, N, \& Oei, T-P (2008). Gambling among the Chinese: A comprehensive review. Clinical Psychology Review, $28(7), 1152-1166$.

Loue, S (1998). Handbook of immigrant health. New York: Plenum Press.

Lund, I (2006). Gambling and problem gambling in Norway: What part does the gambling machine play? [Article]. Addiction Research \& Theory, 14(5), 475-491.

Markus, A, Jupp, J, \& McDonald, P (2009). Australia's Immigration Revolution. Crows Nest, NSW: Allen \& Unwin.

Minister for Immigration and Citizenship. (2007). Important changes to general skilled migration commence today. In Joint media release with the Hon Julie Bishop MP, Minister for Education, Science and Training, 1 September 2007. Retrieved 5 May, 2010, from http://pandora.nla.gov.au/pan/67564/20071110-0000/www.minister.immi.gov.au/ media/media-releases/2007/ka07078-joint.html.

Minister for Immigration and Citizenship. (2010). Migration reforms to deliver Australia's skills needs. Media release 8 February 2010. Retrieved 8 February, 2010, from http://www.minister.immi.gov.au/media/media-releases/2010/ ce10006.htm.

Ministry of Health (2009). A Focus on Problem Gambling: Results of the 2006/07 New Zealand Health Survey. Wellington: New Zealand Government.

Moore, SM, \& Ohtsuka, K (1997). Gambling Activities of Young Australians: Developing a Model of Behaviour. Journal of Gambling Studies, 13(3), 207-236.

Neal, P, Delfabbro, PH, \& O'Neil, M (2005). Problem Gambling and Harm: Towards a National Definition. Melbourne: Report prepared for the National Gambling Research Program Working Party.

Oei, T, \& Raylu, N (2009). The Relationship Between Cultural Variables and Gambling Behavior Among Chinese Residing in Australia. Journal of Gambling Studies, 25(4), 433-445.

Productivity Commission (1999). Australia's Gambling Industries: Inquiry Report No.10. Melbourne: Productivity Commission.

Productivity Commission (2010). Gambling: Productivity Commission Inquiry, Volume 1, Report No.50 (Vol. 1). Canberra: Productivity Commission.

Raylu, N, \& Oei, TP (2004). Role of culture in gambling and problem gambling. Clinical Psychology Review, 23(8), 1087-1114

Scull, S, \& Woolcock, G (2005). Problem gambling in non-English speaking background communities in Queensland, Australia: a qualitative exploration. International Gambling Studies, 5(1), 29-44.

Shepherd, R-M, Ghodse, H, \& London, M (1998). A Pilot Study Examining Gambling Behaviour Before and After the Launch of the National Lottery and Scratch Cards in the UK. Addiction Research \& Theory, 6(1), 5-12.

Stanton, M (2004). Gambling problems: Relationships and social networks: Introduction to the series. Weekly Addiction Gambling Education Report, 9(49), 1-2.

Stevens, M, Golebiowska, K, \& Morrison, P (2010). Correlates of reported gambling problems in the CALD population of Australia (pp. 1-60). Melbourne: Office of Gaming and Racing Department of Justice for Gambling Research Australia. 
Stevens, M, \& Young, M (2009). Reported Gambling Problems in the Indigenous and Total Australian Population. Melbourne: Office of Gaming and Racing, Department of Justice for Gambling Research Australia.

Tanasornnarong, N, Jackson, A, \& Thomas, S (2004). Gambling among young Thai people in Melbourne, Australia: an exploratory study. International Gambling Studies, 4(2), 189-203.

Teo, P, Mythily, S, \& Anantha, S (2007). Demographic and Clinical Features of 150 Pathological Gamblers Referred to a Community Addictions Programme. Annals of the Academy of Medicine, Singapore, 36(3), 165-168.

The Ethnic Communities' Council of New South Wales. (1999). Gambling among members of ethnic communities in Sydney. Sydney: Ethnic Communities' Council of NSW for the Casino Community Benefit Fund.

Toneatto, T, \& Brennan, J (2002). Pathological gambling in treatment-seeking substance abusers. Addictive Behaviors, $27(3), 465-469$.

Volberg, RA (1994). The prevalence and demographics of pathological gamblers: Implications for public health. American Journal of Public Health, 84(2), 237-241.

Westermeyer, J (1999). The role of cultural and social factors in the cause of addictive disorders. The Psychiatric Clinics of North America, 22(2), 253-273.

Winter, N (2008). Programs for Stata Retrieved 19 December, 2008. from http://www.faculty.virginia.edu/nwinter/progs/.

Wohl, MJA, \& Enzle, ME (2002). The Deployment of Personal Luck: Sympathetic Magic and Illusory Control in Games of Pure Chance. Personality and Social Psychology Bulletin, 28(10), 1388-1397.

Wohl, MJA, Young, MM, \& Hart, KE (2005). Untreated young gamblers with game-specific problems: Self-concept involving luck, gambling ecology and delay in seeking professional treatment. Addiction Research \& Theory, 13(5), 445-459.

Young, M, \& Stevens, M (2009). Player preferences and social harm: An analysis of the relationships between player characteristics, gambling modes, and problem gambling. International Journal of Mental Health and Addiction, $7(1), 262-279$.

Young, M, Stevens, M, \& Morris, M (2008). Problem gambling within the non-Indigenous population of the Northern Territory of Australia: A multivariate analysis of risk factors. International Gambling Studies, 8(1), 77-93.

doi:10.1186/2195-3007-3-1

Cite this article as: Stevens and Golebiowska: Gambling problems amongst the CALD population of Australia: hidden, visible or not a problem?. Asian Journal of Gambling Issues and Public Health 2013 3:1.

\section{Submit your manuscript to a SpringerOpen ${ }^{\circ}$ journal and benefit from:}

- Convenient online submission

- Rigorous peer review

- Immediate publication on acceptance

- Open access: articles freely available online

- High visibility within the field

- Retaining the copyright to your article

Submit your next manuscript at $\boldsymbol{\nabla}$ springeropen.com 